\title{
Framework for Safely Reopening Endoscopy During the COVID-19 \\ Pandemic: Redefining Adequate Preparation
}

\begin{abstract}
Hala Fatima, MD; Andrea Shin, MD
Indiana University Health Physicians, Indianapolis, Indiana (Dr Fatima); and Department of Internal Medicine/Gastroenterology, Indiana University School of Medicine Indianapolis, Indiana (Drs Fatima and Shin).

The novel coronavirus disease 2019 (COVID-19) pandemic has had a major impact on outpatient endoscopy practice. Starting mid-March 2020, all elective endoscopic procedures across the country were suspended in order to suppress viral spread and conserve personal protective equipment (PPE) for high acuity care. As most geographic areas in the United States reach their peaks, proceduralists are preparing to reopen for business. This should involve a strategic approach based on individual states' readiness and COVID-19 burden. The timeline and criteria for reopening remain unclear in light of the rapidly evolving data. This article highlights some of these nuances and describes a conceptual framework that will help guide endoscopists in the reopening process.
\end{abstract}

\section{Framework for Reopening}

A prerequisite for resumption of endoscopy is approval by local municipal and state health authorities, ${ }^{1}$ as well as a consistent reduction in new cases per day for 14 days in the geographic area according to the joint guidance document developed by the Centers of Medicare \& Medicaid Services, the American Hospital Association, in partnership with the American College of Surgeons, the American Society of Anesthesiologists, and the Association of periOperative Registered Nurses. The American Medical

This is the author's manuscript of the work published in final edited form as:

Fatima, H., \& Shin, A. (2020). Framework for Safely Reopening Endoscopy During the COVID-19 Pandemic: Redefining Adequate Preparation. Journal of Public Health Management and Practice. https://doi.org/10.1097/PHH.0000000000001235 
Association has published a guide to reopening physician practices $^{2}$; reopening of endoscopy may build upon this guidance, with the addition of special considerations that are unique to endoscopic practice. Consultation with local health departments is essential before endoscopy resumption is contemplated. Bidirectional communication is necessary to provide local agencies with information on case positivity or disease burden within the practice and to receive information about local developments including outbreaks. These steps are critical in ensuring that adequate resources are available to respond to changing needs. In addition, city and state health officials should be involved in the reopening process, so clear plans can be put in place for staff, physicians, and patients.

Factors to consider include facility readiness (including healthy staff), continued observation of social distancing practices, and adequate supply of PPE and sedation medications. On the one hand, there are concerns including delayed patient care, pent-up demand for endoscopy, as well as financial repercussions that push health care systems and physicians to open early; conversely, there is the real threat of viral resurgence among health care workers and the general population. Resumption must be performed in phases, based on clinical priority of cases to enable social distancing and maintaining the PPE supply while not compromising patient care or safety of health care workers.

\section{Case Prioritization}

Risk of delaying endoscopy depends on indications and patient factors. Patient factors (eg, age, comorbidity, and immune status) need consideration to weigh the benefit of endoscopy against the risk of contracting and spreading COVID-19 infection. At the same time, bandage strategies instead of definitive treatment may lead to increase in morbidity and mortality. Establishing a prioritization work group consisting of endoscopists and administrators is preferable to help guide scheduling. A list of previously postponed procedures should be obtained, and cases should be classified into tiers depending on urgency and clinical priority. An example classification scheme is shown as follows ${ }^{3}$ :

- Tier 1 (urgent): Procedures that cannot wait and should be scheduled now; 
- Tier 2 (time-sensitive): Procedures that may be delayed several weeks but are to be prioritized as capacity permits; and

- Tier 3 (elective): Procedures that can be delayed with no long-term clinical impact.

\section{Phases of Endoscopy Resumption}

- Current status: Emergent, urgent, and high priority procedures

- Phase 1: $25 \%$ capacity

- Phase 2: $50 \%$ capacity

- Phase 3: 75\% capacity

- Phase 4: $100 \%$ capacity

It should be kept in mind that despite attempts at reopening, patients' perspectives might drive the schedule or case volumes due to fears around exposure risk. The plan of phased reopening should be shared and discussed with the local city and state health officials whose guidance is key to safe resumption of clinical practices. Governors, state health, and local officials need to be involved to assess whether the proposed plan of phased reopening is appropriate for their communities depending on the changing density of COVID-19 cases in the area. Plans should include the process for assessing the required PPE supply, workflow procedures, monitoring or surveillance of patients and visitors diagnosed with COVID-19, and the plan for how teams will handle (ie, track, report) positive cases for both patients and staff. This approach should be tailored yet flexible and will play a major role in reducing risk of transmission and exposure to patients and to conserve adequate supplies (PPE and medical personnel) during a public health crisis.

\section{Team Manpower and Bandwidth}


During endoscopy unit closures, nurses and technicians are redeployed to other areas of need. Preemptive discussions need to ensue to discuss the timeline of return to their usual roles in endoscopy. If staff were deployed to a COVID-19 unit, then discussions need to include reliable SARS-CoV-2 testing strategies before return to the endoscopy unit for face-to-face patient care. Procedural volume surge should be expected, ${ }^{4}$ given the pent-up need for endoscopy and likely increased need for endoscopy in COVID-19 survivors for gastrointestinal sequelae. It is essential to ensure that trained staff are available to absorb increased procedural volume as we move along the phases of reopening. In addition, tentative plans for weekend endoscopy sessions may be considered.

\section{The New Normal}

COVID-19 is here to stay with periodic resurgences. Needless to say, the current modifications in medical care will need to be long term until herd immunity is established or a vaccine is available.

\section{Screening of patients}

A phone call prior to the scheduled procedure should be performed to screen each patient for symptoms, history of exposure, and/or a positive COVID-19 test. The return-to-work criteria for health care professionals per the Centers for Disease Control and Prevention (CDC) guidelines ${ }^{5}$ can be used for scheduling such patients while also considering the level of urgency for the procedure. Screening should be done for patients coming in for all endoscopic procedures and not just esophagogastroduodenoscopy (EGD), because surface contamination is a cause of infectious spread in addition to aerosol. In addition, in the event of sedation complications such as respiratory arrest, N95 mask is mandated for COVID-19positive and/or symptomatic patients (Figure).

For patients who are asymptomatic or presymptomatic, screening can be done with a non-test-based criteria or test-based criteria. The former is based on symptoms including fever, cough, or shortness of breath and exposure to COVID-19. Reliability is questionable, given the rate of asymptomatic carriers in 
the population with known community spread. These are silent spreaders and hence an important factor for possible resurgence. ${ }^{6,7}$ Current recommendations from the national society organizations are to consider all individuals in areas of community spread to be at "high risk" of SARS-CoV-2 infection exposure. $^{3,8}$

The test-based strategy is better but is limited by accuracy and nonavailability of universal testing. Although test sensitivity of PCR-based diagnostic testing for COVID-19 can be upward of 96\%, there is a wide variation in the clinical sensitivity and negative predictive value due to issues such as operator dependence, suboptimal sampling site, and processing errors; this leads to further gray zones in management. While as positive test result indicates need for additional precautions including use of a negative pressure endoscopy room, a negative test does not definitely rule out the risk of infection transmission. In the case of the latter, PPE would still be indicated. During an outbreak of a highly contagious illness, PPE should include standard precautions with appropriate modifications (ie, respiratory protection). ${ }^{9}$ The turnaround time is variable depending on duration of the test and the laboratory workload; in-house testing may be anywhere from 90 minutes to several hours. Point of care may take 5 minutes for a positive result and 15 minutes for a negative result and is more feasible in the setting of outpatient procedures. Patient compliance for testing may be another limitation to this approach.

\section{Social distancing}

Patient attendants/drivers must be prohibited from entering the building. Social distancing must be observed in the waiting room as well as within the endoscopy unit, both among providers and patients. To that end, only a limited number of patients should be present simultaneously in the assessment and recovery areas. This is variable depending on the layout and capacity of each endoscopy unit. It is preferable that teams, each including a physician, a nurse, a technician, and/or an anesthesiologist, should be organized per day that take care of individual patients from check-in to checkout to minimize cross- 
exposure. Only essential staff should be allowed and learners should be minimized. After recovery, patients should be taken to the entrance for pickup.

\section{Screening of providers}

This can be done using either the nontesting or testing criteria as discussed earlier. Major limitations for the latter approach are testing availability, provider willingness, and optimum interval for testing (weekly vs daily).

\section{PPE for providers}

Staff must be trained on proper technique of donning and doffing PPE. Health care workers in direct contact with patients should use surgical masks; cloth masks are not appropriate. Non-patient-facing workers are okay to wear cloth masks. However, availability of PPE is the critical limitation. Given the high demand of PPE during the pandemic, the supply chains are disrupted and many institutions have been forced to develop strict rules for allocating PPE. The CDC does not explicitly classify EGD as an aerosol-generating procedure (AGP). Endoscopic procedures have been omitted from this list, which is admittedly noncomprehensive. Therefore, most hospital systems may not provide N95 or equivalent respirators to endoscopy units in light of the shortage based on the lack of definitive guidance offered by

the CDC. Multiple endoscopists have been infected with COVID-19 as a result of exposure in endoscopy. Although there are preliminary data by Repici et al, ${ }^{10}$ suggesting that risk of transmission during endoscopy is low, these "low" rates occurred in the context of canceling elective and increasing protective measures including implementation of higher-level PPE. It is important to note that a clustering of cases with infection rates up to $44.4 \%$ in health care workers was noted at 3 centers, the majority $(85.7 \%)$ of which occurred prior to the adaptation of stringent protection and restriction of procedures. While these data reassure us that increased protective measures have helped mitigate clinically relevant COVID-19 infections related to endoscopy, the important takeaway is just that clinically relevant COVID-19 infections may be mitigated by increased protective measures. It is unclear as to whether that exposure 
was secondary to aerosolization versus from contamination of surfaces. As it stands, gastroenterological societies representing expert consensus endorse that EGDs are indeed AGPs and hence require N95 masks for providers. To provide safe working conditions and instill confidence, it is absolutely essential to reinventorize PPE before opening back and ensure a steady supply chain.

Other steps that health care workers can take should include changing into scrubs at work and changing back after their shift to prevent viral spread into the community.

\section{Masks for patients}

These should be recommended for all patients coming in for procedures. Surgical face masks are appropriate for asymptomatic patients. If patient's COVID-19 status is known, then N95 respirators should be considered for the ones who are positive. These obviously have to be taken off during an upper endoscopy.

\section{Air flow of procedure rooms}

Before reopening, it must be ensured that the existing air filters are up to code and air exchange is adequate in the procedure rooms. Coronavirus particle is 80 to $160 \mathrm{~nm}$, and it remains active up to 3 hours in indoor air and 3 to 7 days on surfaces depending on the environmental conditions. ${ }^{11}$ Environmental sampling from an intensive care unit and a general COVID-19 ward at Huoshenshan Hospital in Wuhan, China, recently found the virus to be widely distributed throughout air samples and object surfaces with estimated transmission distances of up to $4 \mathrm{~m} .{ }^{12} \mathrm{It}$ is recommended that ventilation systems switched on continuously in order to remove virus particles out of the building. Recirculation should not be used, and portable HEPA filters are not recommended. Additional precautions include waiting for 30 to 45 minutes before bringing next patient in the room to ensure air clearance of viral particles; however, this is not a

proven approach. Procedures, especially EGD, for COVID-19-positive patients should be done in ample airborne infection isolation rooms that utilize negative pressure. 


\section{Decontamination and reuse of face-filtering respirators}

Although disposable N95 respirators and other (face-filtering respirators) are designed for one-time use, decontamination and subsequent reuse have been proposed as a crisis strategy in the event of a global pandemic and national shortage. Unfortunately, many areas and institutions throughout the country now find themselves in the situation. The CDC has issued limited guidance on the available options for decontamination. Various strategies, including ultraviolet germicidal radiation (UVGI), vaporous hydrogen peroxide (VHP), and moist heat, have been proposed..$^{5}$ In order for any decontamination strategy to be considered, 3 conditions must be met: (1) there should be direct evidence of effectiveness against SARS-CoV-2; (2) the method should effectively eliminate potential nosocomial pathogens; (3) the function and fit of the respirator should not be compromised. In recent weeks, the Food and Drug Administration issued an emergency use authorization for the Battelle Decontamination System ${ }^{13}$ that uses VHP to decontaminate N95 respirators based on the available data, which have shown that VHP penetrates all filter layers, inactivates industry standard biological organisms, and renders SARS-CoV-2 noninfectious without adversely affecting fit for up to 20 cycles. UVGI ${ }^{14}$ has also been shown to be effective in inactivating a wide variety of pathogens including coronaviruses while maintaining fit and filtration; however, it is unlikely to kill all viruses due to shadow effects created by the multiple layers and mask construction. Furthermore, dose and duration of UV exposure are critically important, direct effectiveness against SARS-CoV-2 is less clear, and the number of cycles appear more limited (2-4 cycles). Moist heat appears to cause minimal effect on respirator fit and function and was shown to be effective against $\mathrm{H} 1 \mathrm{~N} 1$, but fewer data are available for the effectiveness against other biological organisms or SARS-CoV-2 specifically.

If opting to implement N95 decontamination in the endoscopy unit, it is essential to recognize that no alternative strategies are currently available, workers are aware that these are strategies for mitigating but not eliminating the potential for infection transmission based on the currently available data, and that appropriate precautions are taken to apply these practices safely. These precautions include proper 
handling of masks before and after use, avoidance if touching the inside of the mask, performing routine seal checks prior to each use, visually inspecting the mask for damage, and safe handling of contaminated PPE that will be sent for reprocessing.

\section{Shortage of sedation medications}

The increased number of patients on ventilator for prolonged periods of time has led to a shortage of medications including lorazepam and fentanyl, which are used for endoscopic sedation. Communication with the pharmacy regarding medication inventory is essential before any plan for reopening is rolled out.

\section{Special Considerations}

\section{General anesthesia}

Availability of ventilators and anesthesia machines are limited depending on each hospital's surge of COVID-19 inpatients on ventilator support. Hence, endoscopies that are done under general anesthesia (eg, endoscopic retrograde cholangiopancreatography, double balloon enteroscopy, and pediatric endoscopy) may face limitation during the phase-in period. Per the CDC, endotracheal intubation is an AGP; therefore, all providers should receive N95 masks as part of their PPE. Endoscopists should wait for 15 to 20 minutes after intubation to enter the room.

\section{Summary}

A lot is still unknown about the pathogenesis of the virus and modes of transmission. The course of the virus and its ultimate impact have yet to unfold. As we reopen endoscopy, careful attention to procedural volume, indications, and safety protocols will be paramount to maintaining high-quality endoscopic services amid a pandemic. Our principles of standard and universal precautions must be upheld to protect our patients and our medical workers, who should not be faulted for choosing to elect a higher standard of safety during times of uncertain risk. Individuals, institutions, organizations, and public health 
departments must work together to create a supportive environment with the common goal of maintaining quality and morale. If success is achieved when opportunity meets preparation, let us plan for success by redefining our meaning of adequate preparation for endoscopy. 
References

1. Gottleib S, McClellan M, Silvis L, Rivers C, Watson C. National coronavirus response: a road map to reopening. American Enterprise Institute Web site. https://www.aei.org/researchproducts/report/national-coronavirus-response-a-road-map-to-reopening. Published March 29, 2020. Accessed April 20, 2020.

2. American Medical Association. COVID-19: a physician practice guide to reopening. https://www-ama-assn-org.proxy.ulib.uits.iu.edu/system/files/2020-06/physican-guidereopening-practices-covid-19.pdf. Updated June 3, 2020. Accessed June 10, 2020.

- $\underline{\text { Cited Here }}$

3. Sultan S, Lim JK, Altayar O, et al. AGA Institute rapid recommendations for gastrointestinal procedures during the COVID-19 pandemic. Gastroenterology. 2020.

doi:10.1053/j.gastro.2020.03.072.

4. Joint statement: roadmap for resuming elective surgery after COVID-19 pandemic. https://www.facs.org//media/files/covid19/joint_statement_resuming_elective_surgery_after_covid19.ashx. Published April 17, 2020. Accessed May 10, 2020.

5. Centers for Disease Control and Prevention. Information for healthcare professionals about coronavirus (COVID-19). https://www.cdc.gov/coronavirus/2019-ncov/hcp. Published 2020. Accessed May 10, 2020.

6. Yu X, Yang R. COVID-19 transmission through asymptomatic carriers is a challenge to containment. Influenza Other Respir Viruses. 2020. doi:10.1111/irv.12743. 
7. Zhang J, Wu S, Xu L. Asymptomatic carriers of COVID-19 as a concern for disease prevention and control: more testing, more follow-up. Biosci Trends. 2020. doi:10.5582/bst.2020.03069.

8. Joint Gastroenterology Society message: COVID-19 use of personal protective equipment. https://webfiles.gi.org/links/media/JOINT_GI_SOCIETY_MESSAGE_PPE_FINAL_04012020. pdf. Published 2020. Accessed June 2, 2020.

9. Carr-Locke DL, Soetikno R, Sharaiha RZ. Whatever happened to universal/standard precautions? Gastrointest Endosc. 2020. doi:10.1016/j.gie.2020.04.001.

10. Repici A, Aragona G, Cengia G, et al. Low risk of COVID-19 transmission in GI endoscopy. Gut. 2020. doi:10.1136/gutjnl-2020-321341.

11. van Doremalen N, Bushmaker T, Morris DH, et al. Aerosol and surface stability of SARSCoV-2 as compared with SARS-CoV-1. N Engl J Med. 2020;382(16):1564-1567.

12. Guo ZD, Wang ZY, Zhang SF, et al. Aerosol and surface distribution of severe acute respiratory syndrome coronavirus 2 in hospital wards, Wuhan, China, 2020. Emerg Infect Dis. 2020;26(7). doi:10.3201/eid2607.200885.

13. Food and Drug Administration. Emergency use authorization. https://www.battelle.org/docs/default-source/commercial-offerings/industry-solutions/battelleeua.pdf. Accessed April 25, 2020.

14. Nebraska Medicine. N95 Filtering facepiece respirator ultraviolet germicidal irradiation (UVGI) process for decontamination and reuse.

https://www.nebraskamed.com/sites/default/files/documents/covid-19/n-95-decon-process.pdf. Accessed April 25, 2020. 


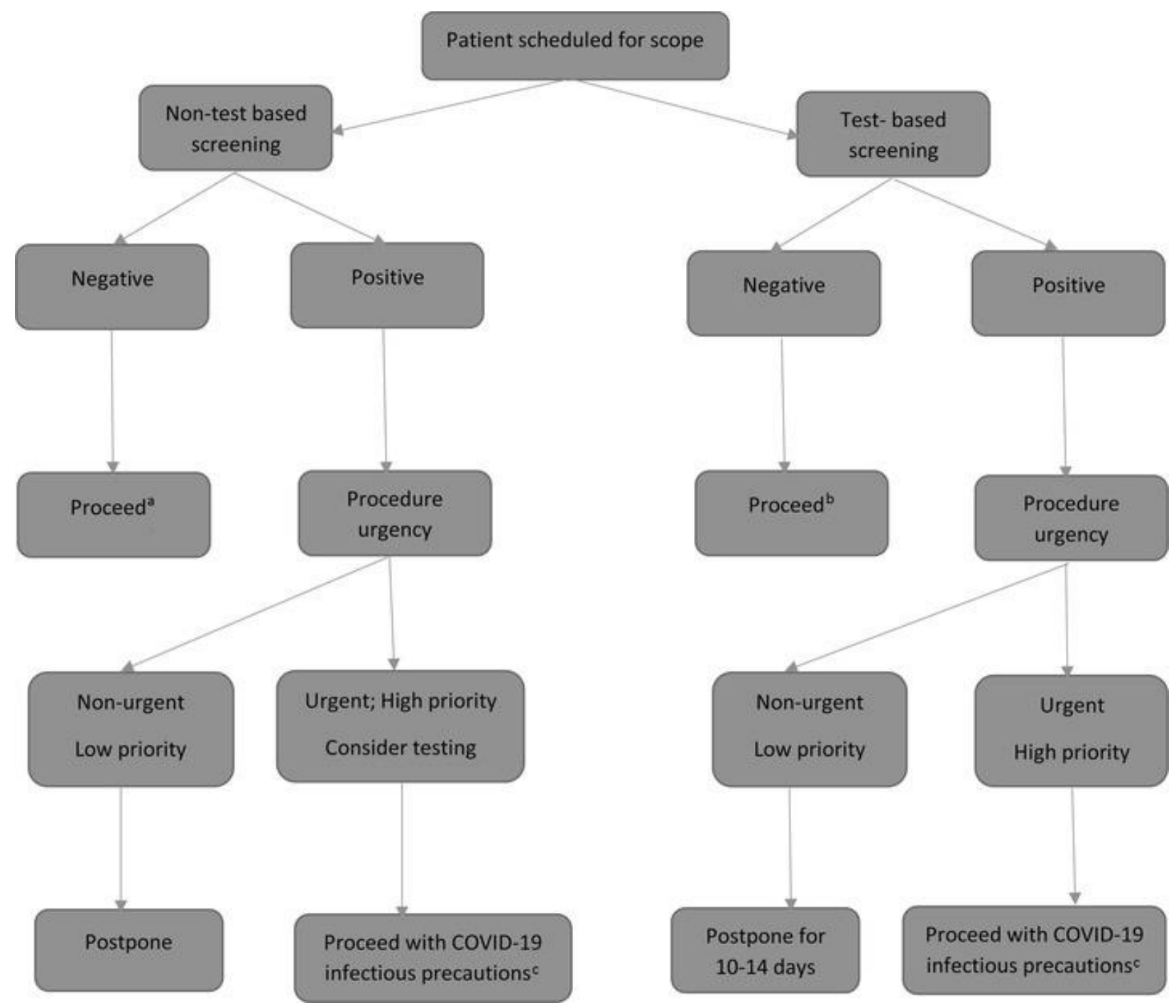

Figure

Suggested Algorithm for Screening Patients for Endoscopy

Abbreviations: EGD, esophagogastroduodenoscopy; PPE, personal protective equipment. ${ }^{\text {PPE }+}$ N95 masks for EGD and standard infectious precautions due to asymptomatic carrier

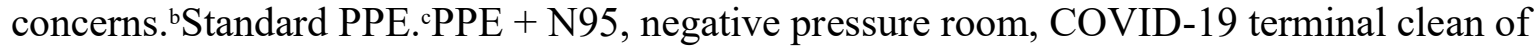
endoscopy room postprocedure (per institution protocol). 